\title{
Some remarks on the inverse eigenvalue problem for real symmetric Toeplitz matrices
}

\author{
N. $\mathrm{Li}^{*}$
}

(Received 8 October 2004, revised 17 November 2005)

\begin{abstract}
A theorem about the bounds of solutions of the Toeplitz Inverse Eigenvalue Problem is introduced and proved. It can be applied to make a better starting generator for iterative numerical methods. This application is tested through a short Mathematica program. Also an optimisation method for solving the Toeplitz Inverse Eigenvalue Problem with a global convergence property is presented. A global convergence theorem is proved.
\end{abstract}

\section{Contents}

\section{Introduction}

C1328

${ }^{*}$ Mathematics Discipline, Faculty of Engineering and Industrial Sciences, Swinburne University of Technology, Melbourne, Australia. mailto:nli@swin.edu.au

See http://anziamj.austms.org.au/V46/CTAC2004/Li for this article, (c) Austral. Mathematical Soc. 2006. Published January 6, 2006. ISSN 1446-8735 
2 Bounds of solutions

C1329

3 A Mathematica program

C1330

4 An optimisation method

C1332

References

C1334

\section{Introduction}

The inverse Toeplitz eigenvalue problem (ToIEP) is to obtain a real vector $\mathbf{r}=\left[r_{1}, r_{2}, \ldots, r_{n}\right]^{t}$ so that the Toeplitz matrix

$$
T(\mathbf{r})=\left[\begin{array}{ccccc}
r_{1} & r_{2} & \cdots & r_{n-1} & r_{n} \\
r_{2} & r_{1} & \cdots & r_{n-2} & r_{n-1} \\
\vdots & \ddots & \ddots & \ddots & \vdots \\
r_{n-1} & r_{n-2} & \cdots & r_{1} & r_{2} \\
r_{n} & r_{n-1} & \cdots & r_{2} & r_{1}
\end{array}\right]
$$

has a prescribed set of real numbers $\left\{\lambda_{1}, \lambda_{2}, \ldots, \lambda_{n}\right\}$ as its spectrum.

Landau [7] proved that every set of $n$ real numbers is the spectrum of an $n \times n$ real symmetric Toeplitz matrix. As the proof is nonconstructive, Newton-type iteration methods are still the main methods to build up such Toeplitz matrices.

The critical task for applying Newton's method is to choose a starting point or an initial approximation properly, otherwise the iterations either diverge or converge to a point which is not a solution. The issue for ToIEP is also mentioned by Laurie [8] and Trench [15]. Theorem 1 in Section 2 gives the bounds of each component of a solution $\mathbf{r}$. Therefore it provides guidance for choosing a starting point. A more reliable starting generator is 
thus produced. A short Mathematica program using this generator is given in Section 3.

There are two categories of iterative methods for solving ToIEP. One [2, 15] exploits the Toeplitz structure while the other $[5,6,8]$ does not. The difference between the two categories is discussed in [1]. All these methods except Trench's do not possess a global convergence property. Trench's method appears to be globally convergent; however, this is not proved. In Section 4 the Levenberg-Marquardt (L-M) method $[13,14]$ with a global convergence feature is presented. The method itself does not need any knowledge of the Toeplitz structure, but its convergence does depend on it.

\section{Bounds of solutions}

Theorem 1 gives the bounds of each component of a solution $\mathbf{r}$.

Theorem 1 If $\mathbf{r}=\left[r_{1}, r_{2}, \ldots, r_{n}\right]^{t}$ is a solution of the ToIEP, then

$$
r_{1}=\sigma_{1} / n
$$

and

$$
\left|r_{i}\right| \leq \sqrt{\frac{n \sigma_{2}-\sigma_{1}^{2}}{2 n(n-i+1)}}, \quad i=2, \ldots, n,
$$

where $\sigma_{k}=\sum_{i=1}^{n} \lambda_{i}^{k}$.

Proof: Equation (2) is well known [15]. Moreover,

$$
\sigma_{2}-\left(\sigma_{1}^{2} / n\right)=\operatorname{trace}\left(T^{2}\right)-n r_{1}^{2}=2 \sum_{i=1}^{n-1} i r_{n+1-i}^{2}
$$


which implies (3), since all terms on the right hand side of (4) are nonnegative. See that for the problem with standardized eigenvalues $\left(\sigma_{1}=0\right.$, $\sigma_{2}=1$ ) [15],

$$
\left|r_{i}\right| \leq \frac{1}{\sqrt{2(n-i+1)}}, \quad i=2, \ldots, n
$$

Theorem 1 gives a clear criterion for selecting an initial approximation when an iterative method is applied. The following well known theorem $[9$, e.g.] follows immediately from the fact that $T(\mathbf{r})$ has the same eigenvalues as the matrix $D^{-1} T(\mathbf{r}) D$, where $D$ is the diagonal matrix whose $i$ th diagonal element is $(-1)^{i+1}$.

Theorem 2 For a given set of real numbers $\left\{\lambda_{1}, \lambda_{2}, \ldots, \lambda_{n}\right\}$, if

$$
\mathbf{r}=\left[r_{1}, r_{2}, \ldots, r_{n}\right]^{t}
$$

is a solution of the ToIEP, then

$$
\tilde{\mathbf{r}}=\left[r_{1},-r_{2}, \ldots,(-1)^{n-1} r_{n}\right]^{t}
$$

is also a solution of the ToIEP.

Theorem 2 shows that the solutions of the ToIEP exist in pairs. It is helpful when we try to locate all possible solutions of the problem.

\section{A Mathematica program}

The starting generator is usually a subtle issue when applying iterative methods. Trench, Laurie and other authors have mentioned the issue for solving ToIEP $[9,15]$. Some generators make a unified starting value for $r_{2}, r_{3}, \ldots, r_{n}$, 
for example $1 / 2(n-1)$, ignoring the differences among these components. Theorem 1 shows that the bounds for $r_{2}$ and $r_{n}$ differ by nearly $\sqrt{n}$ times. When $n$ is large the ignorance will not be acceptable. The short Mathematica program in Algorithm 1 is designed for solving ToIEP which shows how the results of Theorem 1 are used to initiate the subroutine FindRoot. The $i$ th component of a starting point $\mathbf{r}$ is chosen randomly between

$$
\pm 0.5 \sqrt{\frac{n \sigma_{2}-\sigma_{1}^{2}}{2 n(n-i+1)}}
$$

using Random [], which produces a random number between 0 and 1 . The algorithm is quite simple: just solve the equations obtained by equating corresponding coefficients of the characteristic polynomial of $T(\mathbf{r})$ and $P(x)=$ $\left(x-\lambda_{1}\right) \cdots\left(x-\lambda_{n}\right)$. We test the program on a problem with an extremely irregularly clustered spectral data $\{1000,100,99,5,1\}$ which was first presented by Laurie [8].

\section{Algorithm 1:}

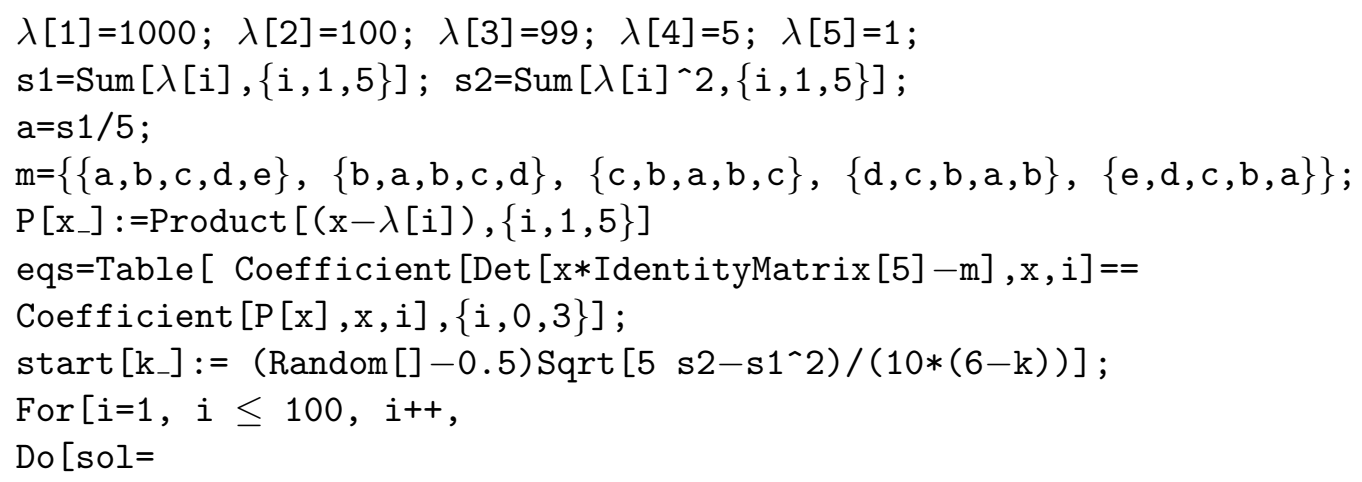

After 100 tries, the following 12 sets of solutions $\left(r_{2}, r_{3}, r_{4}, r_{5}\right)=(b, c, d, e)$ 
with $r_{1}=a=241.000$ were obtained: $\{168.853,212.453,209.583,165.547\}$, $\{-191.89,218.846,-155.583,159.154\},\{-192.256,218.631,-155.536,158.369\}$, $\{168.986,212.011,210.26,164.989\},\{-211.225,169.31,-166.489,211.69\}$, $\{193.838,217.022,152.043,163.978\},\{210.868,168.858,167.156,213.142\}$, $\{-185.502,160.523,-224.893,220.477\},\{193.472,217.237,152.089,164.763\}$, $\{186.977,159.793,224.821,217.207\},\{167.541,210.216,212.945,170.784\}$, $\{167.399,210.668,212.278,171.332\}$.

Actually, from Theorem 2, we have obtained 24 sets of solutions. By changing the sign of $b$ and $d$ of the above sets we get the other 12 sets of the solutions. I expect to obtain more solutions (possibly $5 !=120$ solutions, see $[3,6])$ if we try more times.

\section{An optimisation method}

In the above program the ToIEP is converted to the system of polynomial equations,

$$
f_{i}\left(r_{2}, \ldots, r_{n}\right)=c_{i}\left(r_{2}, \ldots, r_{n}\right)-p_{i}=0, \quad i=2, \ldots, n .
$$

where $c_{i}$ and $p_{i}$ are coefficients of the $\lambda^{n-i}$ term of the characteristic polynomial of $T(r)$ with $r_{1}=\sigma_{1} / n$ and the polynomial $P(x)=\left(x-\lambda_{1}\right) \cdots\left(x-\lambda_{n}\right)$, respectively. We now apply the least squares method to find the solution of the equations. The objective function to be minimised here is

$$
F\left(r_{2}, \ldots, r_{n}\right)=\frac{1}{2} \sum_{i=2}^{n} f_{i}^{2}\left(r_{2}, \ldots, r_{n}\right) .
$$

If at a stage in the minimisation process $F\left(\overline{r_{2}}, \ldots, \overline{r_{n}}\right)=0$, then $\mathbf{r}=$ $\left[r_{1}, \overline{r_{2}}, \ldots, \overline{r_{n}}\right]^{t}$ is a solution of the ToIEP. The Levenberg-Marquardt (LM) method solves this minimisation problem. The L-M method is widely recognized as one of the most reliable methods for nonlinear least squares 
problems. It works extremely well for functions without a high degree of nonlinearity $[10,11,12]$. A hybrid version of the L-M method was developed by Powell [14]. When the elements of the Jacobian of the system of equations are exact, the method has a global convergence property under some conditions. Note that a minimisation program with global convergence property means for any starting point it always converges to either a local minimum or a global minimum, but not always to a global minimum [4]. We state this Powell's result as Theorem 3.

Theorem 3 (Powell) If the functions $f_{i}$ have continuous, bounded first derivatives then the $L-M$ method will finish after a finite number of iterations, due to

$$
F(\mathbf{x})<E
$$

or

$$
F\left(\mathbf{x}^{(k)}\right) \geq M\left\|\mathbf{g}^{(k)}\right\|_{2},
$$

where $E$ and $M$ are assigned fixed positive values before the iterations begin and $\mathbf{g}^{(k)}$ is the gradient vector of $F(x)$ at the $k$ th iterate $\mathbf{x}=\mathbf{x}^{(k)}$.

See that if the iteration terminates due to $F(\mathbf{x})<E$ ( $E$ is a very small number) then $\mathbf{x}$ is approximately a global minimum of the $F(\mathbf{x})$ and is also a solution of $f_{i}=0$; if the iteration stops due to $F\left(\mathbf{x}^{(k)}\right) \geq M\left\|\mathbf{g}^{(k)}\right\|_{2}(M$ is a very large number) $\mathbf{x}^{(k)}$ is approximately a local minimum of $F(\mathbf{x})$. Interestingly, the functions $f_{i}$ of a ToIEP satisfy all conditions of Theorem 3. Thus we have the following theorem:

Theorem 4 Powell's version of $L-M$ method for solving ToIEP has a global convergence property.

Proof: Let $\mathbf{x}=\left(r_{2}, \ldots, r_{n}\right)$ and $\mathbf{x}^{(0)}$ be an initial approximation to the problem. Then the method restricts all iterates $\mathbf{x}^{(k)}$ to the set

$$
S=\left\{\mathbf{x}: F(\mathbf{x}) \leq F\left(\mathbf{x}^{(0)}\right)\right\} .
$$


We claim that $S$ is a compact set. As $F$ is a continuous function $S$ must be closed. Hence we only need to show that $S$ is bounded. It can be shown that

$$
f_{2}=(n-1) r_{2}^{2}+(n-2) r_{3}^{2}+\cdots+r_{n}^{2}-\left(n \sigma_{2}-\sigma_{1}^{2}\right) / 2 n .
$$

Let $c=\sqrt{2 F\left(\mathbf{x}^{(0)}\right)}$, then the inequality $\left|f_{2}\right| \leq c$ gives

$$
\left|r_{i}\right| \leq \sqrt{\frac{n \sigma_{2}-\sigma_{1}^{2}+2 n c}{2 n(n-i+1)}}, \quad i=2, \ldots, n .
$$

Thus $S$ is bounded. Because all the derivatives $f_{i}^{\prime}$ are polynomials on the compact set $S$, they must be continuous and bounded.

\section{References}

[1] Chu, M. T. and Golub, G. H., Structured inverse eigenvalue problems, Acta Numerica, 2001. C1329

[2] Chu, M. T., On a Newton method for the inverse Toeplitz eigenvalue problem. http://www4.ncsu. edu/ ^mtchu/Research/Papers/itep.ps C1329

[3] Garcia, C. B. and Li, T. Y., On the numbers of solutions to polynomial systems of equations, SIAM J. Numer. Anal., 17(4), 1980, 540-546. C1332

[4] Gill, P., Murray, W. and Wright, M., Practical Optimization, page 100, Academic Press, 1981. C1333

[5] Friedland, S., Nocedal, J. and Overton, M. L., The formulaton and analysis of numerical methods for inverse eigenvalue problems, SIAM J. Numer. Anal., 24(3), 1987, 634-667. C1329 
[6] Friedland, S., Inverse eigenvalue problems for symmetric Toeplitz matrices, SIAM J. Matrix Anal. Appl., 13(4), 1992, 1142-1153. C1329, C1332

[7] Landau, H. J., The inverse eigenvalue problem for real symmetric Toeplitz matrices, J. Amer. Math. Soc., 7(3), 1994, 749-767. C1328

[8] Laurie, D. P., A numerical approach to the inverse Toeplitz eigenproblem, SIAM J. Sci. Stat. Comput., 9(2), 1988, 401-405. C1328, C1329, C1331

[9] Laurie, D. P., Initial values for the inverse Toeplitz eigenvalue problem, SIAM J. Sci. Stat. Comput., 22, 2001, 2239-2255. C1330

[10] Li, N., A matrix inverse eigenvalue problem and its application, Linear Algebra And Its Applications, 266, 1997, 143-152. C1333

[11] Li, N., and Chu, K-W. E., Designing the Hopfield neural network via pole assignment, Internat. J. Systems Sci., 25, 1994, 669-681. C1333

[12] Li, N., An Inverse eigenvalue problem and feedback control, In May, R. L., Fitz-Gerald, G. F. and Grundy, I. H., editors, Proceedings of the 4th Biennial Engineering Mathematics and Applications Conference, Melbourne, Australia, pages 183-186. RMIT, 2000. C1333

[13] Osborne, M. R., Nonlinear least squares - the Levenberg-Marquardt algorithm revisited. J. Austral. Math. Soc. Ser. B, 1976, 343-357. C1329

[14] Powell, M. J. D., A hybrid method for nonlinear equations. In P. Rabinowitz, editor, Numerical methods for nonlinear equations, pages 87-114, Gordon and Breach, London, 1970. C1329, C1333

[15] Trench, W. F., Numerical solution of the inverse eigenvalue problem for real symmetric Toeplitz matrices, SIAM J. Sci. Comput., 18(6), 1997, 1722-1736. C1328, C1329, C1330 